\title{
Effect of in vitro testicular spermatozoa culture on pregnancy outcomes: an experience at a single university hospital
}

\author{
Jisun Lee ${ }^{1}$, Jung Hyeon Yoo $^{2}$, Jae Hun Lee ${ }^{3}$, Hyun Soo Ahn ${ }^{4}$, Kyung Joo Hwang ${ }^{3}$, Miran Kim ${ }^{3}$ \\ ${ }^{1}$ Department of Obstetrics and Gynecology, Kyungpook National University Hospital, School of Medicine, Kyungpook National University, \\ Daegu, Korea \\ ${ }^{2}$ Department of Obstetrics and Gynecology, Bundang Jaeseng Hospital, Seongnam, Korea \\ ${ }^{3}$ Department of Obstetrics and Gynecology, Ajou University School of Medicine, Suwon, Korea \\ ${ }^{4}$ Department of Urology, Ajou University School of Medicine, Suwon, Korea
}

Received: September 16, 2020

Revised: November 2, 2020

Accepted: November 5, 2020

Corresponding author:

Miran Kim

Department of Obstetrics and

Gynecology, Ajou University School

of Medicine, 164 Worldcup-ro,

Yeongtong-gu, Suwon 16499, Korea

Tel: $+82-31-219-5250$

Fax: +82-31-219-5245

E-mail:kmr5300@ajou.ac.kr
Background: There are no guidelines for the optimal incubation time or temperature to improve pregnancy outcomes in testicular sperm extraction-intracytoplasmic sperm injection (TESE-ICSI) cycles. We aimed to evaluate whether a 24-hour in vitro culture of testicular spermatozoa affects pregnancy outcomes in TESE-ICSI cycles.

Methods: This was a retrospective study of 83 TESE-ICSI cycles using testicular spermatozoa in 46 couples with male partners suffering from nonobstructive or obstructive azoospermia. Sperm retrieval was performed either on the oocyte retrieval (OR) day (65 cycles in 33 couples; group A) or on the day before OR (18 cycles in 13 couples; group B) followed by in vitro culture for 24 hours. The clinical characteristics and pregnancy outcomes, including the number of retrieved oocytes, fertilization rates, embryo transfer rates, implantation and clinical pregnancy rates, were compared between the two groups.

Results: There were no differences in terms of clinical characteristics except for the levels of luteinizing hormone $(\mathrm{LH})$ in males. Group $B$ had higher $\mathrm{LH}$ levels than group $\mathrm{A}(4.56 \pm 1.24 \mathrm{IU} / \mathrm{L}$ vs. $3.67 \pm 1.07 \mathrm{IU} / \mathrm{L}, p=0.017)$. Group $B$ showed higher fertilization rate $(72.4 \% \pm 32.1 \%$ vs. $59.2 \% \pm 21.7 \%, p=0.045)$, implantation rate $(35.0 \% \pm 34.1 \%$ vs. $14.0 \% \pm 21.5 \%, p=0.010)$, pregnancy rate per cycle $(80 \%$ vs. $39 \%, p=0.033)$, and clinical pregnancy rate per cycle $(80 \%$ vs. $37.5 \%, p=0.024)$ than those of group A.

Conclusion: Testicular sperm retrieval performed on the day before $O R$ followed by in vitro culture can potentially improve pregnancy outcomes.

Keywords: Azoospermia; Intracytoplasmic sperm injections; Testicular sperm retrieval

\section{Introduction}

Almost 20\% of men with infertility suffer from azoospermia [1,2]. Assisted fertilization with testicular sperm extraction (TESE) and intracytoplasmic sperm injection (ICSI) has been successfully ap- plied for the treatment of obstructive and nonobstructive azoospermia. In vivo, spermatozoa are released from the seminiferous tubules and reach epididymal sites where they acquire progressive motility and fertilization potential [3]. However, in TESE cycles, it is difficult to find sufficient number of motile sperms because tes-

Copyright (C) 2021 Yeungnam University College of Medicine

This is an Open Access article distributed under the terms of the Creative Commons Attribution Non-Commercial License (http://creativecommons.org/licenses/by-nc/4.0/) which permits unrestricted non-commercial use, distribution, and reproduction in any medium, provided the original work is properly cited. 
ticular spermatozoa show motility only for a short period of time after testicular biopsy and may become immotile due to depletion of metabolic resources [4].

Sperm motility refers to the movement of sperm and is often used as a clinical parameter to evaluate sperm viability in ICSI cycles. It has been reported that only $3 \%$ of testicular spermatozoa are motile after a biopsy [5]. In vitro culture of spermatozoa has been suggested as a way to improve sperm motility in TESE-ICSI cycles. The efficacy of cryopreserved spermatozoa was first evaluated by Oates et al. [6], who achieved a pregnancy rate of $40 \%$ per couple using cryopreserved spermatozoa. However, studies on in vitro maturation of sperm and its effects on clinical pregnancy outcomes show conflicting results.

Hu et al. [7] reported that sperms extracted on the day before oocyte retrieval (OR) showed increased motility, consequently resulting in a higher fertilization rate. On the contrary, Karacan et al. [8] reported that the timing of TESE does not affect the outcomes of ICSI-embryo transfer (ET) cycles. More importantly, there are no specific guidelines suggesting a suitable incubation time or temperature to improve sperm motility and pregnancy outcomes in TESE-ICSI cycles.

Hosseini and Khalili [9] studied the changes in sperm motility at different time intervals in an in vitro culture of spermatozoa and observed that sperm motility significantly increased after 24 hours but began to decline at day 2 of culture. Therefore, we aimed to evaluate whether a 24-hour in vitro culture of testicular spermatozoa affects outcomes of TESE-ICSI cycles. We compared the clinical characteristics and pregnancy outcomes between TESE-ICSI cycles with fresh testicular spermatozoa obtained on the day of OR and those with in vitro cultured spermatozoa retrieved on the day before OR.

\section{Materials and methods}

\section{Patients}

This was a retrospective study of patients with successful TESE-ICSI cycles with TESE performed either on the day of OR or 24 hours before OR between January 2001 and December 2015 at Ajou University Hospital, Suwon, Korea. Clinical characteristics and pregnancy outcomes were evaluated by reviewing the medical records. This study was approved by the Institutional Review Board (IRB) of Ajou University Hospital (IRB No: AJIRB-MED-MDB-16-038). The informed consent from the patients was waived from the IRB. A total of 83 TESE-ICSI cycles in 46 couples were included in the study. All male partners with obstructive azoospermia or nonobstructive azoospermia, including chemotherapy exposure or varicocele, underwent repeated semen analyses; the absence of sperms on at least two separate semen analyses confirmed the diagnosis of azoospermia.

Clinical characteristics, including male age, female age, type of azoospermia, follicle-stimulating hormone (FSH) level, type of ovulation induction, etiology of infertility, total gonadotropin dose (IU), and pregnancy outcomes, were included in the analysis. Clinical pregnancy was defined as the presence of a gestational sac with fetal heartbeat confirmed by ultrasonography. The implantation rate was analyzed as the number of gestational sacs with fetal heartbeat per embryo transferred.

\section{Ovarian stimulation and oocyte retrieval}

The ovarian stimulation protocol was individualized according to the patients' age, diagnosis, and ovarian reserve. Different stimulation protocols included long gonadotropin-releasing hormone (GnRH) agonist and short GnRH antagonist using recombinant FSH (Follitrope, LG Life Sciences Ltd., Seoul, Korea; Menopur, Ferring Pharmaceuticals, Lausanne, Switzerland), and/or human menopausal gonadotropin (IVF-C, LG Life Sciences Ltd). Transvaginal sonography was used to monitor follicle growth every 2 days until the dominant follicle grew to approximately $16-18 \mathrm{~mm}$. Ovulation was triggered by injecting 10,000 IU of human chorionic gonadotropin (hCG; Pregnyl, Merck \& Co. Inc., Haarlem, the Netherlands) intramuscularly when the mean diameter of the leading follicle reached $\geq 18 \mathrm{~mm}$ or when urine luteinizing hormone (LH) was positive.

Thirty-six hours after hCG administration, OR was performed through vaginal ultrasonography-guided punctures of the ovarian follicles. Following the OR, patients received either intramuscular injection of progesterone (Sugest, 50 mg; Sanzyme Ltd., Hyderabad, India) once a day or vaginal progesterone (Utrogestan, 200 mg; Besins Healthcare Ltd., London, UK) for luteal support until 2 weeks after the procedure when the pregnancy was confirmed by analyzing serum $\mathrm{hCG}$, and continued to receive the injection thereafter.

\section{Testicular sperm extraction and in vitro culture of spermatozoa}

Testicular sperm extraction was performed by a urology specialist either on the day of OR or 24 hours before the day of OR. After sterilization of the scrotal skin, $1 \%$ lidocaine was injected locally into the underlying tunics, and an incision was made to remove a 3-mm segment of testicular tissues. The initial examination for the presence of spermatozoa was performed under an inverted phase-contrast microscope (Diaphot 300/N88, Nikon, Tokyo, Japan). Individual spermatozoa were observed carefully with a high magnification under the microscope to evaluate any sign of sperm 
motility, such as twitching of the head or tail. The suspension was then transferred to a test tube if spermatozoa were noticed. The biopsy samples were then independently minced using sterile needles and dissected into small pieces in a sterile Petri dish.

TESE spermatozoa were accompanied by red blood cells (RBCs), debris, testicular cells, and a large number of dead or immotile spermatozoa that have detrimental effects on existing motile spermatozoa. The biopsy tissues were then centrifuged with an RBC lysis buffer for 5 minutes at $300 \times g$ to eliminate these cells. In group A (TESE performed on the day of OR), the tissue was centrifuged twice at $300 \times g$ for 5 minutes, and the pellet was placed in the Sydney IVF fertilization medium (Cook Medical, Bloomington, IN, USA) containing $10 \%$ human serum albumin. In group B (TESE performed on the day before OR), the extracted tissues were incubated in the same IVF fertilization medium and cultured for 24 hours at $37^{\circ} \mathrm{C}$ in $6 \% \mathrm{CO}_{2}$ and $5 \% \mathrm{O}_{2}$ balance. Centrifugation of the biopsy samples in group $\mathrm{B}$ was performed on the day of the $\mathrm{OR}$. The $\mathrm{pH}$ and temperature of the culture medium and incubator were controlled daily and maintained throughout the study.

\section{Intracytoplasmic sperm injection procedure}

Using sterile 23-gauge needles, the testicular spermatozoa were prepared for oocyte insemination by squeezing the biopsy tissues to identify the tubules and release spermatozoa. The tubules were placed in Quinn's Sperm Washing Medium (SAGE In Vitro Fertilization, Trumbull, CT, USA) containing gentamicin and $5.0 \mathrm{mg} /$ $\mathrm{mL}$ human serum albumin. One drop of sperm washing medium was replaced with a $7 \%$ polyvinylpyrrolidone medium, and five drops were replaced with the sperm washing medium. Motile spermatozoa with forward progression were transferred from the sperm suspension drop to the polyvinylpyrrolidone drop using an injection needle with an inside diameter of 12 to $15 \mu \mathrm{m}$. The spermatozoa were then immobilized and stored in the polyvinylpyrrolidone drop. After this, assisted fertilization by ICSI was performed as previously described [10].

\section{Evaluation of the outcomes of intracytoplasmic sperm injection}

Fertilization was assessed 17 to 19 hours after ICSI, and embryo cleavage and quality were evaluated 2 to 5 days after OR. The criteria for embryo grading were based on the gross morphological appearance on light microscopy [11]. The zygotes were cultured in the media (cleavage medium 10\% human serum albumin; Cook Medical) under oil in a $6 \% \mathrm{CO}_{2}$ and $5 \% \mathrm{O}_{2}$ incubator. The embryos were transferred into Pronase solution (Sigma-Aldrich, St. Louis, MO, USA) under oil for 60 seconds to soften the zona pellucida and then transferred to fresh cleavage medium. Depending on the status of the embryo, ET was performed 3 to 5 days following OR. The number of embryos transferred was determined by the Korean Ministry of Health and Welfare, depending on the female partner's age and embryo development. Embryos with multiple nuclei or cytoplasmic abnormalities were discarded. Progesterone was administered either intramuscularly (Sugest, $50 \mathrm{mg}$ ) once a day or vaginally (Utrogestan, $200 \mathrm{mg}$ ) for luteal support until 2 weeks after the procedure when the pregnancy was confirmed by analyzing serum hCG and continued thereafter.

Clinical pregnancy was defined as the presence of a gestational sac with a fetal heartbeat confirmed by ultrasonography. The implantation rate was analyzed as the ratio between the number of gestational sacs and the number of transferred embryos. The delivery rate was defined as the ratio between the number of deliveries and the number of ETs.

\section{Statistics}

Quantitative variables are described using means and standard deviations, and qualitative variables are shown as percentages. The data were first tested for normality, and then the differences between groups were compared using an independent $t$-test or Mann-Whitney $U$ test depending on the distribution. Pearson chi-square test or Fisher exact test was used to determine the association between two categorical variables. Data were analyzed using IBM SPSS version 25.0 (IBM Corp., Armonk, NY, USA). A p-value of less than 0.05 was considered statistically significant.

\section{Results}

Group A consisted of 65 cycles in 33 patients and group B consisted of 18 cycles in 13 patients. The clinical characteristics of both groups are shown in Table 1. The two groups were comparable in terms of age of male and female partners, basal FSH and anti-Müllerian hormone levels, and the number of stimulation days. The distribution of type of azoospermia, etiology of infertility, and type of ovulation induction also showed no difference between the two groups. Although statistically insignificant, the total dose of gonadotropin used for group B was slightly higher than that used for group A $(2,387.5 \pm 500.3 \mathrm{IU}$ vs. $2,130 \pm 490.4 \mathrm{IU}, p=0.053)$.

Table 2 describes the ICSI outcomes and pregnancy outcomes of groups A and B. The numbers of retrieved oocytes and good-quality oocytes were not significantly different between the two groups ( $16.8 \pm 11.3$ vs. $14.5 \pm 7.6, p=0.608 ; 14.7 \pm 9.7$ vs. $12.4 \pm 6.7$, $p=0.235)$. However, the fertilization rate was significantly higher in group B than in group A $(72.4 \% \pm 32.1 \%$ vs. $59.2 \% \pm 21.7 \%$, $p=0.045)$. Group $B$ also showed higher implantation rate $(35.0 \% \pm 34.1 \%$ vs. $14.0 \% \pm 21.5 \%, p=0.010)$, pregnancy rate per 
Table 1. Clinical characteristics in groups A and B

\begin{tabular}{|c|c|c|c|}
\hline Variable & Group A & Group B & $p$-value \\
\hline No. of cycle/couple & $65 / 33$ & $18 / 13$ & \\
\hline \multicolumn{4}{|l|}{ Age (yr) } \\
\hline Female & $31.1 \pm 3.4$ & $31.1 \pm 3.2$ & $0.925^{b)}$ \\
\hline Male & $34.3 \pm 3.8$ & $35.6 \pm 6.4$ & 0.284 \\
\hline \multicolumn{4}{|l|}{ Basal FSH (IU/L) } \\
\hline Female & $5.3 \pm 1.7$ & $4.2 \pm 2.2$ & 0.304 \\
\hline Male & $4.8 \pm 2.9$ & $4.3 \pm 1.6$ & 0.966 \\
\hline AMH in females $(\mathrm{ng} / \mathrm{mL})^{\mathrm{a})}$ & $4.2 \pm 2.5$ & $4.7 \pm 2.7$ & 0.657 \\
\hline Basal LH in males (IU/L) & $3.6 \pm 1.1$ & $4.6 \pm 1.2$ & 0.017 \\
\hline Type of azoospermia & & & 0.658 \\
\hline Obstructive & 58 (89.2) & 16 (88.9) & \\
\hline Nonobstructive & $7(10.8)$ & $1(5.6)$ & \\
\hline \multicolumn{4}{|l|}{ Etiology of infertility } \\
\hline Only male factor & $42(64.6)$ & $11(61.1)$ & 1.000 \\
\hline Male factor+anovulation & $1(1.5)$ & $1(5.6)$ & $0.389^{c}$ \\
\hline Male factor+tubal factor & $4(6.1)$ & 0 & 0.572 \\
\hline Male factor+endometriosis & $13(20.0)$ & $5(27.8)$ & 0.524 \\
\hline \multicolumn{4}{|l|}{ Type of ovulation induction } \\
\hline $\mathrm{FSH}+\mathrm{GnRH}$ antagonist & $14(21.5)$ & $2(11.1)$ & 0.502 \\
\hline $\mathrm{FSH}+\mathrm{GnRH}$ agonist & $15(23.1)$ & $8(44.4)$ & 0.084 \\
\hline Pure FSH & $8(12.3)$ & $3(16.7)$ & 0.697 \\
\hline $\mathrm{FSH}+\mathrm{hMG}$ & $28(43.1)$ & $5(27.8)$ & 0.286 \\
\hline Total gonadotropin dose (IU) & $2,130.0 \pm 490.4$ & $2,387.5 \pm 500.3$ & 0.053 \\
\hline Duration of stimulation (day) & $8.6 \pm 4.1$ & $9.0 \pm 1.2$ & 0.336 \\
\hline
\end{tabular}

Values are presented as number only, mean \pm standard deviation, or number (\%).

Group A, testicular sperm extraction (TESE) performed on the day of oocyte retrieval (OR); group B, TESE performed on the day before OR.

FSH, follicle-stimulating hormone; LH, luteinizing hormone; AMH, anti-Müllerian hormone; GnRH, gonadotropin-releasing hormone; hMG, human menopausal gonadotropin.

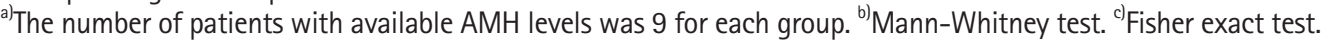

cycle ( $80 \%$ vs. $39 \%, p=0.033)$, and clinical pregnancy rate per cycle ( $80 \%$ vs. $37.5 \%, p=0.024)$ than those of group A. The incidence of miscarriage, ectopic pregnancy, and live birth and cryopreservation rates were comparable between the two groups.

\section{Discussion}

In the present study, we compared the outcomes of TESE-ICSI cycles with fresh spermatozoa obtained on the day of OR and TESE-ICSI cycles with spermatozoa obtained 24 hours before OR. The number of retrieved oocytes, fertilized eggs, and cleaved embryos were comparable between the two groups. On the other hand, TESE-ICSI cycles with spermatozoa obtained 24 hours before OR showed higher fertilization rate, implantation rate, pregnancy rate, and clinical pregnancy rate than those of TESE-ICSI cycles with fresh spermatozoa. These results indicate that in vitro maturation of spermatozoa for 24 hours resulted in improved pregnancy outcomes compared to fresh spermatozoa.
There are various factors known to influence pregnancy outcomes following TESE-ICSI cycles, such as paternal age, maternal age, male testosterone, sperm motility, and/or type of azoospermia [12-14]. Among these factors, the motility of spermatozoa is emphasized as an important predictive factor for successful fertilization by ICSI [15]. In vitro maturation of spermatozoa has been suggested as a way to improve sperm motility, thereby improving pregnancy outcomes in TESE-ICSI cycles [16-19]. In a study by Balaban et al. [20], the fertilization rate increased from $42.1 \%$ to $68.8 \%$ after in vitro culture of spermatozoa with improved sperm motility. Similarly, in our study, group B showed a higher fertilization rate than group A ( $72.4 \%$ vs. $59.2 \%, p=0.045)$ due to in vitro maturation of spermatozoa. The exact mechanism behind improved testicular sperm motility after in vitro culture is not yet clear. Some studies have suggested that co-culture with all testicular cells, including Sertoli, Leydig, and germ cells, may enhance sperm maturation [4]. In addition, the washing procedure before the culture also eliminates inhibitory factors, which activates the 
Table 2. Pregnancy outcomes of TESE-ET cycles in groups $A$ and $B$

\begin{tabular}{|c|c|c|c|}
\hline Variable & Group A & Group B & $p$-value \\
\hline No. of oocytes retrieved & $14.5 \pm 7.6$ & $16.8 \pm 11.3$ & $0.608^{\mathrm{a})}$ \\
\hline No. of good oocytes & $12.4 \pm 6.7$ & $14.7 \pm 9.7$ & 0.235 \\
\hline No. of fertilized eggs & $7.0 \pm 3.7$ & $10.7 \pm 8.8$ & $0.211^{\mathrm{a})}$ \\
\hline Fertilization rate (\%) & $59.2 \pm 21.7$ & $72.4 \pm 32.1$ & 0.045 \\
\hline No. of embryos cleaved & $5.1 \pm 2.1$ & $8.1 \pm 7.0$ & $0.102^{a)}$ \\
\hline No. of good embryos & $2.8 \pm 1.5$ & $2.1 \pm 1.3$ & 0.097 \\
\hline No. of embryos transferred & $3.8 \pm 1.5$ & $2.5 \pm 1.4$ & 0.001 \\
\hline Implantation rate (\%) & $14.0 \pm 21.5$ & $35.0 \pm 34.1$ & $0.010^{\mathrm{a})}$ \\
\hline Pregnancy rate per cycle & 25 (38.5) & $12(66.7)$ & 0.033 \\
\hline Clinical pregnancy rate per cycle & $24(36.9)$ & $12(66.7)$ & 0.024 \\
\hline No. of miscarriages & $4(6.2)$ & $1(5.6)$ & $1.000^{b)}$ \\
\hline No. of ectopic pregnancies & $1(1.5)$ & 0 & $1.000^{b)}$ \\
\hline No. of of live births & $13(20.0)$ & $2(11.1)$ & 0.040 \\
\hline Cryopreservation rate & $18(27.7)$ & $9(50.0)$ & 0.074 \\
\hline
\end{tabular}

Values are presented as mean \pm standard deviation or number (\%).

Group A, testicular sperm extraction (TESE) performed on the day of oocyte retrieval (OR); group B, TESE performed on the day before OR.

a) Mann-Whitney test. ${ }^{\text {b) }}$ Fisher exact test.

essential factors required for enhanced motility [12]. However, a long-term culturing may decrease sperm motility because of the gradual degeneration of testicular somatic cells.

Despite collective evidence to support sperm retrieval before OR and have sperm cultured before ICSI, there are no guidelines to suggest suitable incubation times. Determination of appropriate sperm retrieval timing and incubation times before the ICSI procedure is critical. Various incubation time periods have been suggested as an optimal time to improve sperm motility and pregnancy outcomes. Wu et al. [21] observed that after a 24-hour in vitro culture, the number of motile sperm showed a remarkable increase and reached a maximum motility rate between 48 and 72 hours. Hu et al. [7] also investigated the feasibility of performing testicular biopsies 24 hours prior to $\mathrm{OR}$ and observed that after in vitro culture, the overall fertilization rate was $58 \%$, the implantation rate was $20 \%$, and the clinical pregnancy rate was $45 \%$. In another study, Hosseini and Khalili [9] observed that sperm motility changed from $13 \%$ immediately after biopsy to $76 \%$ at 24 hours and $15 \%$ at 72 hours of culture, suggesting that 1 day of culture is an ideal in vitro culture time to optimize sperm motility in azoospermic TESE samples. However, the results of these studies only implied that in vitro culture may enhance sperm motility but failed to provide evidence for the effect on pregnancy outcomes.

Levran et al. [18] first reported the outcomes of TESE-ICSI using testicular spermatozoa obtained on the day of OR or the day before OR. They obtained similar fertilization rates $(61.7 \%$ vs. $58.9 \%$ ) and clinical pregnancy rates (34.8\% vs. $29.2 \%)$ in their analysis of 47 IVF-ICSI cycles using testicular spermatozoa retrieved on the day of or on the day before oocyte aspiration [18].
More recently, Karacan et al. [8] compared the fertilization rates between ICSI-ET cycles with fresh testicular spermatozoa obtained on the same day or the day before OR with frozen-thawed spermatozoa. They observed no difference between the groups in terms of pregnancy outcomes with a fertilization rate of $68.7 \%$ $70 \%$, implantation rate of $13.4 \%-16.5 \%$, and clinical pregnancy rate of $30.9 \%-31.3 \%$.

Contrary to the results of Karacan et al. [8], our study showed significantly higher implantation rate, pregnancy rate per cycle, and clinical pregnancy rate per cycle in ICSI cycles using in vitro cultured spermatozoa than in those with fresh spermatozoa (35.0\% vs. $14.0 \%, p=0.010 ; 80 \%$ vs. $39 \%, p=0.033 ; 80 \%$ vs. $37.5 \%, p=0.024$, respectively). In the study by Karacan et al. [8], the baseline characteristics between the two groups were not different. On the contrary, group A in our study included a higher proportion of female infertility patients along with azoospermic male partners than group $\mathrm{B}$. This may have influenced group $\mathrm{B}$ to have a higher number of retrieved oocytes and fertilized eggs, consequently leading to improved pregnancy outcomes compared to those of group A.

Another possible explanation may be the different hormone levels of male partners between the two groups. Although statistically insignificant, group A had higher basal FSH levels than group B $(4.8 \pm 2.9 \mathrm{IU} / \mathrm{L}$ vs. $4.3 \pm 1.67 \mathrm{IU} / \mathrm{L}, p=0.966)$. The baseline $\mathrm{LH}$ levels in group $\mathrm{B}$, however, were higher than those in group $\mathrm{A}$ $(4.56 \pm 1.24 \mathrm{IU} / \mathrm{L}$ vs. $3.67 \pm 1.07 \mathrm{IU} / \mathrm{L}, p=0.017)$. There have been conflicting reports on the association between hormonal levels and the chance of sperm retrieval. Salehi et al. [22] reported that high levels of FSH are significantly associated with lower chances of sperm retrieval in azoospermic men. On the other 
hand, some studies have shown that serum FSH concentration is not an independent predictive factor for sperm retrieval because FSH concentration is controlled by various endocrine and paracrine factors $[23,24]$. The discrepancies in male FSH and LH levels observed in our study might have resulted from the small number of the study population in group B. If the number of cycles were equivalent between the two groups, the baseline hormonal levels could have different values. Nonetheless, our data suggest that the baseline male hormone levels might have influenced the pregnancy outcomes in TESE-ICSI cycles.

Our study has several limitations. The uneven sample size of groups $\mathrm{A}$ and $\mathrm{B}$ makes it difficult to extrapolate significant statistical importance. Moreover, although we have shown that fertilization rate, implantation rate, and pregnancy rates were significantly improved with in vitro cultured spermatozoa, we are unable to provide data for any change in sperm motility before and after in vitro culture. Any changes in sperm motility were only observed by the laboratory technician, and the recorded data were not provided. Another limitation is the effect of different controlled ovarian hyperstimulation $(\mathrm{COH})$ protocols on pregnancy outcomes. It is known that different $\mathrm{COH}$ protocols may influence pregnancy outcomes. Because we aimed to evaluate the effect of in vitro culture of spermatozoa on pregnancy outcomes and the number of each $\mathrm{COH}$ protocol for groups $\mathrm{A}$ and $\mathrm{B}$ was too small, we did not compare the pregnancy outcomes according to the $\mathrm{COH}$ protocol. Nevertheless, our study provides enough evidence to support the previous findings that in vitro culture of spermatozoa in TESE-ICSI cycles may significantly improve pregnancy outcomes. Further studies with large sample sizes are needed to make definitive statements in this regard.

In conclusion, a sperm extraction procedure can be performed on the day before egg retrieval without compromising the risk of pregnancy. Although the mechanism is yet unknown, the in vitro culture of sperm before OR may improve clinical outcomes, especially for patients undergoing TESE-ICSI for azoospermia.

\section{Acknowledgments}

\section{Conflicts of interest}

No potential conflict of interest relevant to this article was reported.

\section{Author contributions}

Conceptualization: JL, JHY, HSA, KJH, MK; Data curation: JL, JHL, HSA, KJH; Formal analysis: JL, JHL, KJH, MK; Funding acquisition, Validation: JHY; Resources: JL, HSA, KJH, MK; Supervision: $\mathrm{KJH}, \mathrm{MK}$; Writing-original draft: JL; Writing-review \& ed- iting: JHY, KJH, MK.

\section{ORCID}

Jisun Lee, https://orcid.org/0000-0002-2870-3176

Jung Hyeon Yoo, https://orcid.org/0000-0002-7593-7581

Jae Hun Lee, https://orcid.org/0000-0003-2103-8078

Hyun Soo Ahn, https://orcid.org/0000-0002-9764-2004

Kyung Joo Hwang, https://orcid.org/0000-0001-7073-9124

Miran Kim, https://orcid.org/0000-0001-5553-5334

\section{References}

1. Coetzee K, Ozgur K, Berkkanoglu M, Bulut H, Isikli A. Reliable single sperm cryopreservation in Cell Sleepers for azoospermia management. Andrologia 2016;48:203-10.

2. Bocca S, Moussavi V, Brugh V, Morshedi M, Stadtmauer L, Oehninger S. ICSI outcomes in men undergoing TESE for azoospermia and impact of maternal age. Andrologia 2017;49: e12617.

3. Moore $\mathrm{HD}$, Akhondi MA. In vitro maturation of mammalian spermatozoa. Rev Reprod 1996;1:54-60.

4. Angelopoulos T, Adler A, Krey L, Licciardi F, Noyes N, McCullough A. Enhancement or initiation of testicular sperm motility by in vitro culture of testicular tissue. Fertil Steril 1999; $71: 240-3$

5. Lacham-Kaplan O, Trounson A. The effects of the sperm motility activators 2-deoxyadenosine and pentoxifylline used for sperm micro-injection on mouse and human embryo development. Hum Reprod 1993;8:945-52.

6. Oates RD, Lobel SM, Harris DH, Pang S, Burgess CM, Carson RS. Efficacy of intracytoplasmic sperm injection using intentionally cryopreserved epididymal spermatozoa. Hum Reprod 1996;11:133-8.

7. Hu Y, Maxson WS, Hoffman DI, Ory SJ, Licht MR, Eager S. Clinical application of intracytoplasmic sperm injection using in vitro cultured testicular spermatozoa obtained the day before egg retrieval. Fertil Steril 1999;72:666-9.

8. Karacan M, Alwaeely F, Erkan S, Çebi Z, Berberoğlugil M, Batukan M, et al. Outcome of intracytoplasmic sperm injection cycles with fresh testicular spermatozoa obtained on the day of or the day before oocyte collection and with cryopreserved testicular sperm in patients with azoospermia. Fertil Steril 2013; 100:975-80.

9. Hosseini A, Khalili MA. Improvement of motility after culture of testicular spermatozoa: the effects of incubation timing and temperature. Transl Androl Urol 2017;6:271-6.

10. Bechoua S, Berki-Morin Y, Michel F, Girod S, Sagot P, Fauque P. 
Outcomes with intracytoplasmic sperm injection of cryopreserved sperm from men with spinal cord injury. Basic Clin Androl 2013;23:14.

11. Scott RT Jr, Hofmann GE, Veeck LL, Jones HW Jr, Muasher SJ. Embryo quality and pregnancy rates in patients attempting pregnancy through in vitro fertilization. Fertil Steril 1991;55: 426-8.

12. Schwarzer JU, Fiedler K, v Hertwig I, Krüsmann G, Würfel W, Schleyer M, et al. Sperm retrieval procedures and intracytoplasmatic spermatozoa injection with epididymal and testicular sperms. Urol Int 2003;70:119-23.

13. Park YS, Lee SH, Lim CK, Choi HW, An JH, Park CW, et al. Paternal age as an independent factor does not affect embryo quality and pregnancy outcomes of testicular sperm extraction-intracytoplasmic sperm injection in azoospermia. Andrologia 2018;51:e13187.

14. Meijerink AM, Cissen M, Mochtar MH, Fleischer K, Thoonen I, de Melker AA, et al. Prediction model for live birth in ICSI using testicular extracted sperm. Hum Reprod 2016;31:1942-51.

15. Hessel M, Robben JC, D’Hauwers KW, Braat DD, Ramos L. The influence of sperm motility and cryopreservation on the treatment outcome after intracytoplasmic sperm injection following testicular sperm extraction. Acta Obstet Gynecol Scand 2015;94:1313-21.

16. Zhu J, Tsirigotis M, Pelekanos M, Craft I. In-vitro maturation of human testicular spermatozoa. Hum Reprod 1996;11:231-2.

17. Liu J, Tsai YL, Katz E, Compton G, Garcia JE, Baramki TA. Outcome of in-vitro culture of fresh and frozen-thawed human testicular spermatozoa. Hum Reprod 1997;12:1667-72.

18. Levran D, Ginath S, Farhi J, Nahum H, Glezerman M, Weiss- man A. Timing of testicular sperm retrieval procedures and in vitro fertilization-intracytoplasmic sperm injection outcome. Fertil Steril 2001;76:380-3.

19. Windt ML, Coetzee K, Kruger TF, Menkveld R, van der Merwe JP. Intracytoplasmic sperm injection with testicular spermatozoa in men with azoospermia. J Assist Reprod Genet 2002;19: 53-9.

20. Balaban B, Urman B, Sertac A, Alatas C, Aksoy S, Mercan R, et al. In-vitro culture of spermatozoa induces motility and increases implantation and pregnancy rates after testicular sperm extraction and intracytoplasmic sperm injection. Hum Reprod 1999;14:2808-11.

21. Wu B, Wong D, Lu S, Dickstein S, Silva M, Gelety TJ. Optimal use of fresh and frozen-thawed testicular sperm for intracytoplasmic sperm injection in azoospermic patients. J Assist Reprod Genet 2005;22:389-94.

22. Salehi P, Derakhshan-Horeh M, Nadeali Z, Hosseinzadeh M, Sadeghi E, Izadpanahi MH, et al. Factors influencing sperm retrieval following testicular sperm extraction in nonobstructive azoospermia patients. Clin Exp Reprod Med 2017;44:22-7.

23. Bryson CF, Ramasamy R, Sheehan M, Palermo GD, Rosenwaks Z, Schlegel PN. Severe testicular atrophy does not affect the success of microdissection testicular sperm extraction. J Urol 2014;191:175-8.

24. Ramasamy R, Padilla WO, Osterberg EC, Srivastava A, Reifsnyder JE, Niederberger C, et al. A comparison of models for predicting sperm retrieval before microdissection testicular sperm extraction in men with nonobstructive azoospermia. J Urol 2013;189:638-42. 